Www.mjn.mosuljournals.com

\title{
Effectiveness of an Education Program on Nurses knowledge concerning in Nursing Management for patients with Third degree and bundle branch block in Kirkuk Teaching Hospitals
}

Article information

Article history:

Received January 3, 2019

Accepted March 7, 2019

Available online June 12, 2019

\author{
DOI: $10.33899 / \mathrm{mjn} .2019 .160084$ @2020, College of Nursing, University of Mosul. \\ Creative Commons Attribution 4.0 International License \\ https://mjn.mosuljournals.com/article $160084 . \mathrm{html}$ \\ Abid Salih Kumait AL-Jumaily ${ }^{1}$ \\ Khalidah Mohammed Khudur ${ }^{2}$
}

\begin{abstract}
Background and Objectives: Heart block is a disease or acquired condition that causes a fault within the heart's natural pacemaker due to some kind of obstruction (or "block") in the electrical conduction system of the heart.Nurses play an important role in maintaining the health and well-being of patients. Aims of the study was to assess the effectiveness of nursing education program on nursing knowledge concerning in nursing management toward third degree and bundle branch block in Kirkuk's teaching hospitals and to identifying the relationship between the nurses' knowledge and their demographic characteristics such as (age, gender and education level)

Material and Method :A descriptive study design (quasi experimental study )was carried out at Azadi teaching hospital and Kirkuk general hospital in Kirkuk city for nursing staff who worked at CCU ,RCU unit, emergency unit and internal medicine unit ,from February up to May, 2018. A non probability (purposive) sample was selected and composed of (80) Nurses (male and female) .Nurses was divided into two groups, study group consisted of (40) nurses exposed to the nursing educational program and control group consisted of (40) nurses were not exposed to the program from Azadi Teaching Hospital and Kirkuk General Hospital in Kirkuk city. The program and instruments were constructed by the researcher for the purpose of the study. The questionnaire was constructed for the purpose of the study which consisted of three parts: the first part include the demographic data (5 ) items (age, gender,residencr, Educational achievement and marital status. The second about training in cardiac care (2) items ( work place and name of training in cardiac care. The third part consist from (18) items of general knowledge toward management of third degree of heart block and bundle branch block of heart block. The (SPSS) ver. (22.0) was used for data analysis. Mean, SD, and ANCOVA test, compare means were used to analyze the collected data. Level of significance was the threshold at $\mathrm{p}<0.05$
\end{abstract}

\footnotetext{
${ }^{1}$ Lectrer, Department of Adult Nursing, College of Nursing, University of Kirkuk Email: abid_master2014@uokirkuk.edu.iq.

${ }^{2}$ Ass. Professor, Head of Department of Adult Nursing, College of Nursing, University of Baghdad
} 
Results: There were 40 nurses included in the study. Relative to "Gender" 23(57.5\%) from sample were female are more illustrated, since, also the study shows the high percent of nursese were at age between (20-39 years) and constitute 35(87.5\%) and most of "Age Groups", are focused at the first two groups since.$(72.5 \%)$.Also the results shows $(65.0 \%)$ were female. With regard to residence $28(70.0 \%)$ were living in urban areas, The study findings indicated that there were highly significant differences between pre and post tests in the study group in overall main domains related to nurses' knowldg abou management of third degree heart block and bundle branch block

Conclusion: The study concluded that the effectiveness of educational program regarding nurses' nurses' knowldg abou management of third degree heart block and bundle branch block .

Keywords: Education Program , nursing management, Heart Block

\section{Introduction}

Atrioventricul (AV) heart block is marked by a disturbance in electrical impulse conduction from the atria to the ventricles. Depending on the type of AV block, the disturbance may be insignificant or it could lead to potentially fatal arrhythmias. AV blocks are classified as firstdegree, second-degree, and third-degree. Firstdegree $\mathrm{AV}$ blocks are the least concerning; third-degree blocks are the most dangerous (Reynolds, 2014 ) .

Cardio Vascular Disease remains a major healthcare problem and one of the most consumers of the public health resources. Heart block (HB) and Ischaemic heart diseases (IHD) remain the commonest cause of death all over the world. As in statistical reports of the World Health Organization (WHO); 2011, the rate of death per 100,000 due to coronary artery diseases in Yemen was 238.5, Sudan; 212.0, Bangladesh; 203.7, Libya; 199.3 and Jordan; 162.5 .(WHO,2011).

Despite the lack of accurate epidemiology data for AVB, it is clear that it is not uncommon in both apparently healthy populations and those with overt heart disease. Approximately $1 \%$ to $2 \%$ of normal subjects have first-degree AVB which increases to $5 \%$ in men over the age of 60 with cardiac diseases (Crisel and Farzaneh,2011)

The prevalence of second-degree AVB for Mobitz II block is estimated to be $3 \%$ in patients with $\mathrm{HF}$, and it is estimated that $5 \%$ to $10 \%$ of people will develop a third-degree heart block in those $>70$ years old and having a history of heart disease ( Chow and Marine,2012).

Atrioventricular (AV) block is a common complication of acute Myocardial Infarction (MI). In pre-thrombolytic era, high (second or third degree) AV block was seen in approximately $5-7 \%$ of patients presenting with acute MI.1 In setting of Inferior MI, this was even as high as $28 \%$ (Rathore et al ,2015)

The prevalence of first-degree atrioventricular block in the general population is approximately $4 \%$, and it is associated with an increased risk of atrial fibrillation. Cardiac pacing for any indication in patients with firstdegree heart block is associated with worse 


\section{Mosul Journal of Nursing, Vol. 9, No. 1, 2021 ( 8-20 )}

outcomes compared with patients with normal atrioventricular conduction. Among patients with heart failure, first-degree atrioventricular block is present in anywhere between $15 \%$ and $51 \%$. Data from cardiac resynchronization therapy studies have shown that first-degree atrioventricular block is associated with an increased risk of mortality and heart failure hospitalization. Recent studies suggest that optimization of atrioventricular delay in patients with cardiac resynchronization therapy is an important target for therapy; however, the optimal method for atrioventricular resynchronization remains unknown. Understanding the role of first-degree atrioventricular block in the treatment of patients with heart failure will improve medical and device therapy. (ACCF,2016)

There are currently more than 3 million patients worldwide with implanted permanent pacemaker. In Europe, Japan and USA, the implantation rate is almost 300-1000 per million. In United States, the prevalence of third-degree AV block is $0.02 \%$. Worldwide, the prevalence of third-degree AV block is $0.04 \%$. The incidence of AV conduction abnormalities increases with advancing age. (Stewart et al ,2011)

In Iraq and by the experience of the researcher the study is important because there is no evidence based research about the level of nurses knowldge to ward heart block inKirkuk city there for the researcher decided to study this issue. The objectives of the study were to assess the effectiveness of nursing education program on nursing knowledge concerning in nursing management toward third degree and bundle branch block in Kirkuk's teaching hospitals and to identifying the relationship between the nurses' knowledge and their demographic characteristics.

\section{MATERIALS AND METHOD}

To achieve the objectives of the study A descriptive study design was conducted on nurses staff who work at Azadi Teaching Tospitals and kirkuk Teaching Hospitals from February up to May, 2018.A nonprobability (purposive) sample of (40) of Nurses staff whom work at Cardiac care unit(CCU), Respiratory Care Unit(RCU), Emergency unit and internal medicine (male and female). The selection of nurses was simply randomly, who ethical approval informed consent was obtained from each participant included in this study. The validity of the questionneir was determined through presenting it to (20) specialist experts and its reliability was determined through using Cronbach's alpha coefficient. Finally, the test-retest reliability of the knowledge questionnaire and 10 nurses were assessed twice in four-week intervals. For the purpose of data collection, a questionnaire was used, which consists

of three parts, first part concerning the demographic data form that included the(age, gender,residencr, Educational achievement and marital status, The second part about training in cardiac care (2) items ( work place and name of training in cardiac care .The third part concerning in Nursing knowledge toward management of heart block .The questionnaire consistsof 23 items 


\section{Mosul Journal of Nursing, Vol. 9, No. 1, 2021 ( 8-20 )}

covering (3) domains includes (general Nurses knowledge towarement of third degree heart block of (7) multiple questions, Nurses knowledge concerning in managementt of bundle branch block heart block and cosist from (8) multiple questions and Nurses knowledge concerning in drugs and pacemaker used in management of $\mathrm{AV}$ heart block this part consist of( 8) multiple questions related to drugs and pacemaker used in management of AV heart block and medications. The study was implemented at Azadi Teaching hospitals and kirkuk Teaching

\section{Analysis Data and result}

Table (1): Distribution of the studied groups according to socio-demographical characteristics variables (SDCv.) (SDCv.) with comparisons significant(No .40)
Hospitals. The nurses who met the study criteria, were approached in the study, the nurses were invited to participate in the study and explain the study objectives. The data analysis through use a descriptive statistical analysisprocedures and inferential analysis procedures (SPSS 22.0) .The data were analyzed through the application of descriptive statistical analysis which include (frequency and percentage, mean of score) and inferential statistical analysis which include (ANCOVA) test .

\begin{tabular}{|c|c|c|c|c|c|c|}
\hline \multirow{2}{*}{ SDCv. } & \multirow{2}{*}{$\begin{array}{l}\text { Groups } \\
\text { Classes }\end{array}$} & \multicolumn{2}{|c|}{ Study } & \multicolumn{2}{|c|}{ Control } & \multirow{2}{*}{ C.S. ${ }^{(*)}$} \\
\hline & & No. & $\%$ & No. & $\%$ & \\
\hline \multirow{3}{*}{ Gender } & Male & 17 & 42.5 & 15 & 37.5 & \multirow{3}{*}{$\begin{array}{c}\text { C.C. }=0.051 \\
P=0.648 \\
\text { (NS) }\end{array}$} \\
\hline & Female & 23 & 57.5 & 25 & 62.5 & \\
\hline & Total & 40 & 100 & 40 & 100 & \\
\hline \multirow{5}{*}{ Age Group } & $20-29$ & 26 & 65.0 & 17 & 42.5 & \multirow{5}{*}{$\begin{array}{c}\text { C.C. }=0.287 \\
P=0.066 \\
(\text { NS) }\end{array}$} \\
\hline & $30-39$ & 9 & 22.5 & 20 & 50.0 & \\
\hline & $40-49$ & 4 & 10.0 & 3 & 7.50 & \\
\hline & $50-59$ & 1 & 2.50 & $\mathbf{0}$ & $\mathbf{0 . 0 0}$ & \\
\hline & Total & 40 & 100 & 40 & 100 & \\
\hline \multirow{3}{*}{ Residence } & Urban & 28 & 70.0 & 31 & $\mathbf{7 7 . 5}$ & \multirow{3}{*}{$\begin{array}{c}\text { C.C. }=0.220 \\
P=0.131 \\
(N S)\end{array}$} \\
\hline & Rural & 12 & 30.0 & 9 & 22.5 & \\
\hline & Total & 40 & 100 & 40 & 100 & \\
\hline \multirow{4}{*}{ Marital status } & Single & 26 & 65.0 & 16 & 40.0 & \multirow{4}{*}{$\begin{array}{c}\text { C.C. }=\mathbf{0 . 2 4 4} \\
\text { P }=0.088 \\
\text { (NS) }\end{array}$} \\
\hline & Married & 12 & 30.0 & 20 & 50.0 & \\
\hline & Divorced & 2 & 5.0 & 4 & 100.0 & \\
\hline & Total & 40 & 100 & 40 & 100 & \\
\hline \multirow{4}{*}{$\begin{array}{c}\text { Educational } \\
\text { achievement } \\
\text { (Nursing) }\end{array}$} & Preparatory Nursing & 6 & 15.0 & 7 & $\mathbf{1 7 . 5}$ & \multirow{4}{*}{$\begin{array}{c}\text { C.C. }=0.108 \\
\begin{array}{c}\text { P=0.621 } \\
\text { (NS) }\end{array}\end{array}$} \\
\hline & Nursing Institute & 14 & 35.0 & 10 & 25.0 & \\
\hline & Bachelor and more & 20 & 50.0 & 23 & 57.5 & \\
\hline & Total & 40 & 100 & 40 & 100 & \\
\hline
\end{tabular}

${ }^{(*)}$ NS: Non Sig. at P>0.05; Testing based on a contingency coefficient (C.C.) test.

Relative to "Gender" subjects are from female are more illustrated, since 23(57.5\%), and 25(62.5\%) are accounted in the study and controlled groups respectively, and most of "Age Groups", are focused at the first two groups since 35(87.5\%), and $37(92.5 \%)$ are accounted in the study and controlled groups respectively, as well as most of residential subjects, are focused at urban area, since $28(70.0 \%)$, and 31(77.5\%) are accounted in the study and controlled groups respectively.With regard to marital status, shows that single subjects has recorded the most od study group, while married status has recorded the most of studied subjects in the controlled group, and they are accounted 26(65.0\%), and 20(50.0\%) respectively. Finally, educational achievement of studied 


\section{Mosul Journal of Nursing, Vol. 9, No. 1, 2021 ( 8-20 )}

subjects shows that most of them has graduate institute, and bachelor or more degrees, and they are accounted 34(85.0\%), and 33(82.5\%) in the study and controlled groups respectively.

Table (2): Distribution of the studied groups according to training in cardiac care indicators with comparisons significant

\begin{tabular}{|c|c|c|c|c|c|c|}
\hline \multirow{2}{*}{ Training } & \multirow{2}{*}{$\begin{array}{c}\text { Groups } \\
\text { Classes } \\
\end{array}$} & \multicolumn{2}{|c|}{ Study } & \multicolumn{2}{|c|}{ Control } & \multirow{2}{*}{ C.S. ${ }^{\left({ }^{*}\right)}$} \\
\hline & & No. & $\%$ & No. & $\%$ & \\
\hline \multirow{6}{*}{ Work place } & CCU Unit & 15 & 37.5 & 14 & 35.0 & \multirow{6}{*}{$\begin{array}{c}\text { C.C. }=0.105 \\
P=0.926 \\
(N S)\end{array}$} \\
\hline & Medical Ward & 8 & 20.0 & 8 & 20.0 & \\
\hline & Emergency Unit & 6 & 15.0 & 5 & 12.5 & \\
\hline & ECG Unit & 2 & 5.00 & 1 & 2.50 & \\
\hline & RCU Unit & 9 & 22.5 & 12 & 30.0 & \\
\hline & Total & 40 & 100 & 40 & 100 & \\
\hline \multirow{4}{*}{ Name of training } & $\mathrm{CCU}$ & 21 & 52.5 & 22 & $\mathbf{5 5 . 0}$ & \multirow{4}{*}{$\begin{array}{c}\text { C.C. }=\mathbf{0 . 0 5 9} \\
\text { P=0.871 } \\
\text { (NS) }\end{array}$} \\
\hline & RCU & 15 & 37.5 & 13 & 32.5 & \\
\hline & ECG & 4 & 10.0 & 5 & 12.5 & \\
\hline & Total & 40 & 100 & 40 & 100 & \\
\hline
\end{tabular}

${ }^{(*)}$ NS: Non Sig. at P>0.05; Testing based on a contingency coefficient (C.C.) test.

Table (2) indicate that the majority of studied subjects are worked at CCU unit, since they are accounted $15(37.5 \%)$, and 14(35.0\%) in the study and controlled groups respectively, as well as the most of training named concerned with the studied subjects previously was at CCU unit, since they are accounted $21(52.5 \%)$, and $22(55.0 \%)$ in the study and controlled groups respectively.

Table (3): Descriptive Statistics of the studied groups according to (Nurses knowledge concerning in management of Third degree heart block (Td)) along studied periods with comparisons significant

\begin{tabular}{|c|c|c|c|c|c|c|c|c|c|c|c|c|c|c|}
\hline \multirow{2}{*}{$\begin{array}{c}\text { Nurses knowledge } \\
\text { concerning in } \\
\text { management of Third } \\
\text { degree heart block (Td) }\end{array}$} & \multirow[b]{2}{*}{ Period } & \multirow[b]{2}{*}{$\begin{array}{l}\mathbf{N} \\
\mathbf{0}\end{array}$} & \multicolumn{6}{|c|}{ Study } & \multicolumn{6}{|c|}{ Control } \\
\hline & & & MS & $\begin{array}{l}\text { S } \\
\mathbf{D}\end{array}$ & $\begin{array}{l}\mathbf{R} \\
\mathbf{S} \\
\%\end{array}$ & \begin{tabular}{l|l|} 
A \\
ss \\
$\cdot$
\end{tabular} & $\begin{array}{l}\text { Co } \\
\text { m. }\end{array}$ & $\begin{array}{l}\text { C. } \\
\text { S. }\end{array}$ & $\begin{array}{c}\mathbf{M} \\
\mathbf{S}\end{array}$ & $\begin{array}{l}\text { S } \\
\mathbf{D}\end{array}$ & $\begin{array}{l}\mathbf{R} \\
\mathbf{S} \\
\%\end{array}$ & $\begin{array}{c}\text { Ass } \\
\text {. }\end{array}$ & $\begin{array}{l}\mathbf{C} \\
\mathbf{o} \\
\mathbf{m}\end{array}$ & C.S. \\
\hline \multirow{3}{*}{$\begin{array}{c}\text { Third-degree AV heart } \\
\text { block is also called }\end{array}$} & Pre & 40 & 0.18 & $\begin{array}{l}0 . \\
38\end{array}$ & $\begin{array}{r}18 \\
.0\end{array}$ & $\mathbf{M}$ & $\begin{array}{l}1 \\
X \\
2\end{array}$ & $\begin{array}{l}\mathbf{H} \\
\mathbf{S}\end{array}$ & $\begin{array}{l}\mathbf{0 .} \\
33\end{array}$ & $\begin{array}{l}0 . \\
47\end{array}$ & $\begin{array}{c}33 . \\
0\end{array}$ & $\mathbf{L}$ & $\begin{array}{l}1 \\
X \\
2\end{array}$ & NS \\
\hline & Post1 & 40 & 0.88 & $\begin{array}{l}\mathbf{0 .} \\
33\end{array}$ & $\begin{array}{c}88 \\
.0\end{array}$ & $\mathbf{H}$ & $\begin{array}{l}1 \\
\mathbf{X} \\
\mathbf{3}\end{array}$ & $\begin{array}{l}\mathbf{H} \\
\mathbf{S}\end{array}$ & $\begin{array}{l}\mathbf{0 .} \\
22\end{array}$ & $\begin{array}{l}0 . \\
42\end{array}$ & 22. & $\mathbf{L}$ & $\begin{array}{l}1 \\
X \\
3\end{array}$ & NS \\
\hline & Post2 & 40 & 0.90 & $\begin{array}{l}\text { 0. } \\
30\end{array}$ & $\begin{array}{l}90 \\
.0\end{array}$ & $\mathbf{H}$ & $\begin{array}{l}\mathbf{2} \\
\mathbf{X} \\
\mathbf{3}\end{array}$ & $\begin{array}{l}\mathbf{N} \\
\mathbf{S}\end{array}$ & $\begin{array}{l}0 . \\
22\end{array}$ & $\begin{array}{l}0 . \\
42\end{array}$ & $\begin{array}{c}22 . \\
0\end{array}$ & $\mathbf{L}$ & $\begin{array}{l}2 \\
\mathbf{X} \\
\mathbf{3}\end{array}$ & NS \\
\hline \multirow{3}{*}{$\begin{array}{l}\text { A third degree heart } \\
\text { block is best } \\
\text { characterized by }\end{array}$} & Pre & 40 & 0.13 & $\begin{array}{l}\mathbf{0 .} \\
33\end{array}$ & $\begin{array}{r}13 \\
.0\end{array}$ & $\mathbf{M}$ & $\begin{array}{l}1 \\
X \\
2\end{array}$ & $\begin{array}{l}\mathbf{H} \\
\mathbf{S}\end{array}$ & $\begin{array}{l}0 . \\
08\end{array}$ & $\begin{array}{l}\mathbf{0 .} \\
27\end{array}$ & $\begin{array}{c}7.5 \\
0\end{array}$ & $\mathbf{L}$ & $\begin{array}{l}1 \\
X \\
2\end{array}$ & NS \\
\hline & Post1 & 40 & 0.85 & $\begin{array}{l}0 . \\
36\end{array}$ & $\begin{array}{l}85 \\
.0\end{array}$ & $\mathbf{H}$ & $\begin{array}{l}1 \\
\mathbf{X} \\
\mathbf{3}\end{array}$ & $\begin{array}{l}\mathbf{H} \\
\mathbf{S}\end{array}$ & $\begin{array}{l}0 . \\
08\end{array}$ & $\begin{array}{l}\mathbf{0 .} \\
27\end{array}$ & $\begin{array}{c}7.5 \\
0\end{array}$ & $\mathbf{L}$ & $\begin{array}{l}1 \\
\mathbf{X} \\
\mathbf{3}\end{array}$ & $\mathbf{S}$ \\
\hline & Post2 & 40 & 0.85 & $\begin{array}{l}0 . \\
36\end{array}$ & $\begin{array}{l}85 \\
.0\end{array}$ & $\mathbf{H}$ & $\begin{array}{l}2 \\
X \\
3\end{array}$ & $\begin{array}{l}\mathbf{N} \\
\mathbf{S}\end{array}$ & $\begin{array}{l}0 . \\
18\end{array}$ & $\begin{array}{l}0 . \\
38\end{array}$ & $\begin{array}{c}18 . \\
0\end{array}$ & $\mathbf{L}$ & $\begin{array}{l}\mathbf{2} \\
\mathbf{X} \\
\mathbf{3}\end{array}$ & $\mathbf{S}$ \\
\hline
\end{tabular}




\begin{tabular}{|c|c|c|c|c|c|c|c|c|c|c|c|c|c|c|}
\hline \multirow[b]{2}{*}{$\begin{array}{l}\text { Nurses knowledge } \\
\text { concerning in } \\
\text { management of Third } \\
\text { degree heart block (Td) }\end{array}$} & \multirow[b]{2}{*}{ Period } & \multirow[b]{2}{*}{$\begin{array}{l}\mathbf{N} \\
\mathbf{o}\end{array}$} & \multicolumn{6}{|c|}{ Study } & \multicolumn{6}{|c|}{ Control } \\
\hline & & & MS & $\begin{array}{l}\text { S } \\
\mathbf{D}\end{array}$ & $\begin{array}{l}\mathbf{R} \\
\mathbf{S} \\
\%\end{array}$ & $\begin{array}{l}\text { A } \\
\text { s } \\
\text { s. }\end{array}$ & $\begin{array}{c}\text { C } \\
\text { o } \\
\text { m. }\end{array}$ & $\begin{array}{l}\text { C. } \\
\text { S. }\end{array}$ & $\begin{array}{c}\mathbf{M} \\
\mathbf{S}\end{array}$ & $\begin{array}{l}\text { S } \\
\mathbf{D}\end{array}$ & $\begin{array}{l}\mathbf{R} \\
\mathbf{S} \\
\%\end{array}$ & $\begin{array}{c}\text { As } \\
\text { s. }\end{array}$ & $\begin{array}{l}\mathbf{C} \\
\mathbf{o} \\
\mathbf{m}\end{array}$ & C.S. \\
\hline \multirow{3}{*}{$\begin{array}{l}\text { In third degree AV } \\
\text { heart block both } \\
\text { atrium and ventricle } \\
\text { work }\end{array}$} & Pre & 40 & 0.35 & $\begin{array}{c}0 . \\
48\end{array}$ & \begin{tabular}{|c|}
$\mathbf{3 5}$ \\
.0
\end{tabular} & $\mathbf{M}$ & $\begin{array}{l}1 \\
\mathbf{X} \\
\mathbf{2}\end{array}$ & $\begin{array}{l}\mathbf{H} \\
\mathbf{S}\end{array}$ & $\begin{array}{c}0 . \\
25\end{array}$ & $\begin{array}{c}0 . \\
44\end{array}$ & $\begin{array}{r}25 \\
.0\end{array}$ & $\mathbf{L}$ & $\begin{array}{l}1 \\
\mathbf{X} \\
\mathbf{2}\end{array}$ & NS \\
\hline & Post1 & 40 & 0.75 & $\begin{array}{l}0 . \\
44\end{array}$ & $\begin{array}{c}75 \\
.0\end{array}$ & $\mathbf{H}$ & $\begin{array}{l}\mathbf{1} \\
\mathbf{X} \\
\mathbf{3}\end{array}$ & $\begin{array}{l}\mathbf{H} \\
\mathbf{S}\end{array}$ & $\begin{array}{c}0 . \\
28\end{array}$ & $\begin{array}{c}0 . \\
45\end{array}$ & $\begin{array}{r}28 \\
.0\end{array}$ & $\mathbf{L}$ & $\begin{array}{l}\mathbf{1} \\
\mathbf{X} \\
\mathbf{3}\end{array}$ & NS \\
\hline & Post2 & 40 & 0.75 & $\begin{array}{l}0 . \\
44\end{array}$ & $\begin{array}{c}75 \\
.0\end{array}$ & $\mathbf{H}$ & $\begin{array}{l}\mathbf{2} \\
\mathbf{X} \\
\mathbf{3}\end{array}$ & $\begin{array}{l}\mathbf{N} \\
\mathbf{S}\end{array}$ & $\begin{array}{c}\text { 0. } \\
35\end{array}$ & $\begin{array}{l}0 . \\
48\end{array}$ & $\begin{array}{c}35 \\
.0\end{array}$ & $\mathbf{M}$ & $\begin{array}{l}\mathbf{2} \\
\mathbf{X} \\
\mathbf{3}\end{array}$ & NS \\
\hline \multirow{3}{*}{$\begin{array}{l}\text { Third degree heart } \\
\text { block is alethal } \\
\text { dysrhythmia because it } \\
\text { may progress to }\end{array}$} & Pre & 40 & 0.32 & $\begin{array}{c}0 . \\
47\end{array}$ & $\begin{array}{c}\mathbf{3 2} \\
.0\end{array}$ & $\mathbf{M}$ & $\begin{array}{l}1 \\
\mathbf{X} \\
\mathbf{2}\end{array}$ & $\begin{array}{l}\mathbf{H} \\
\mathbf{S}\end{array}$ & $\begin{array}{c}0 . \\
10\end{array}$ & $\begin{array}{c}\text { 0. } \\
\text { 30 }\end{array}$ & $\begin{array}{r}10 \\
.0\end{array}$ & $\mathbf{L}$ & $\begin{array}{l}1 \\
\mathbf{X} \\
\mathbf{2}\end{array}$ & NS \\
\hline & Post1 & 40 & 0.75 & $\begin{array}{l}0 . \\
44\end{array}$ & $\begin{array}{r}75 \\
.0\end{array}$ & $\mathbf{H}$ & $\begin{array}{l}\mathbf{1} \\
\mathbf{X} \\
\mathbf{3}\end{array}$ & $\begin{array}{c}\mathbf{H} \\
\mathbf{S}\end{array}$ & $\begin{array}{c}0 . \\
10\end{array}$ & $\begin{array}{c}\text { 0. } \\
30\end{array}$ & $\begin{array}{r}10 \\
.0\end{array}$ & $\mathbf{L}$ & $\begin{array}{l}\mathbf{1} \\
\mathbf{X} \\
\mathbf{3}\end{array}$ & $\mathbf{S}$ \\
\hline & Post2 & 40 & 0.83 & $\begin{array}{c}0 . \\
38\end{array}$ & $\begin{array}{r}83 \\
.0\end{array}$ & $\mathbf{H}$ & $\begin{array}{l}2 \\
\mathbf{X} \\
\mathbf{3}\end{array}$ & $\begin{array}{l}\mathbf{N} \\
\mathbf{S}\end{array}$ & $\begin{array}{c}0 . \\
23\end{array}$ & $\begin{array}{c}0 . \\
42\end{array}$ & $\begin{array}{r}23 \\
.0\end{array}$ & $\mathbf{L}$ & $\begin{array}{l}2 \\
X \\
3\end{array}$ & $\mathbf{S}$ \\
\hline \multirow{3}{*}{$\begin{array}{c}\text { With third degree AV } \\
\text { heart block the atrial } \\
\text { rate is }\end{array}$} & Pre & 40 & 0.18 & $\begin{array}{c}0 . \\
38\end{array}$ & $\begin{array}{c}18 \\
.0\end{array}$ & $\mathbf{M}$ & $\begin{array}{l}1 \\
\mathbf{X} \\
\mathbf{2}\end{array}$ & $\begin{array}{l}\mathbf{H} \\
\mathbf{S}\end{array}$ & $\begin{array}{c}0 . \\
13\end{array}$ & $\begin{array}{c}\text { 0. } \\
33\end{array}$ & $\begin{array}{r}13 \\
.0\end{array}$ & $\mathbf{L}$ & $\begin{array}{l}1 \\
X \\
2\end{array}$ & NS \\
\hline & Post1 & 40 & 0.83 & $\begin{array}{c}0 . \\
38\end{array}$ & \begin{tabular}{|c|}
83 \\
.0
\end{tabular} & $\mathbf{H}$ & $\begin{array}{l}\mathbf{1} \\
\mathbf{X} \\
\mathbf{3}\end{array}$ & $\begin{array}{l}\mathbf{H} \\
\mathbf{S}\end{array}$ & $\begin{array}{c}0 . \\
10\end{array}$ & $\begin{array}{c}\text { 0. } \\
30\end{array}$ & $\begin{array}{r}10 \\
.0\end{array}$ & $\mathbf{L}$ & $\begin{array}{l}\mathbf{1} \\
\mathbf{X} \\
\mathbf{3}\end{array}$ & NS \\
\hline & Post2 & 40 & 0.90 & $\begin{array}{c}\text { 0. } \\
\mathbf{3 0}\end{array}$ & $\begin{array}{c}90 \\
.0\end{array}$ & $\mathbf{H}$ & $\begin{array}{l}2 \\
\mathbf{X} \\
\mathbf{3}\end{array}$ & $\begin{array}{l}\mathbf{N} \\
\mathbf{S}\end{array}$ & $\begin{array}{c}0 . \\
20\end{array}$ & $\begin{array}{c}0 . \\
41\end{array}$ & $\begin{array}{r}20 \\
.0\end{array}$ & $\mathbf{L}$ & $\begin{array}{l}2 \\
X \\
3\end{array}$ & $\mathbf{S}$ \\
\hline \multirow{3}{*}{$\begin{array}{c}\text { Nursing management } \\
\text { of third degree heart } \\
\text { block is }\end{array}$} & Pre & 40 & 0.15 & $\begin{array}{c}0 . \\
36\end{array}$ & $\begin{array}{c}15 \\
.0\end{array}$ & $\mathbf{M}$ & $\begin{array}{l}1 \\
\mathbf{X} \\
\mathbf{2}\end{array}$ & $\begin{array}{c}\mathbf{H} \\
\mathbf{S}\end{array}$ & $\begin{array}{c}0 . \\
13\end{array}$ & $\begin{array}{c}\text { 0. } \\
33\end{array}$ & $\begin{array}{r}13 \\
.0\end{array}$ & $\mathbf{L}$ & $\begin{array}{l}1 \\
\mathbf{X} \\
\mathbf{2}\end{array}$ & NS \\
\hline & Post1 & 40 & 0.88 & $\begin{array}{c}\text { 0. } \\
33\end{array}$ & $\begin{array}{c}88 \\
.0\end{array}$ & $\mathbf{H}$ & $\begin{array}{l}\mathbf{1} \\
\mathbf{X} \\
\mathbf{3}\end{array}$ & $\begin{array}{c}\mathbf{H} \\
\mathbf{S}\end{array}$ & $\begin{array}{c}\text { 0. } \\
05\end{array}$ & $\begin{array}{c}0 . \\
22\end{array}$ & $\begin{array}{c}5 . \\
00\end{array}$ & $\mathbf{L}$ & $\begin{array}{l}1 \\
\mathbf{X} \\
\mathbf{3}\end{array}$ & NS \\
\hline & Post2 & 40 & 0.95 & $\begin{array}{c}0 . \\
22\end{array}$ & $\begin{array}{c}95 \\
.0\end{array}$ & $\mathbf{H}$ & $\begin{array}{l}\mathbf{2} \\
\mathbf{X} \\
\mathbf{3}\end{array}$ & $\begin{array}{l}\mathbf{N} \\
\mathbf{S}\end{array}$ & $\begin{array}{c}0 . \\
18\end{array}$ & $\begin{array}{c}0 . \\
38\end{array}$ & $\begin{array}{r}18 \\
.0\end{array}$ & $\mathbf{L}$ & $\begin{array}{l}2 \\
X \\
3\end{array}$ & $\mathbf{S}$ \\
\hline
\end{tabular}

${ }^{(*)}$ HS: Highly Sig. at $\mathrm{P}<0.01$; S: Sig. at $\mathrm{P}<0.05$; NS: Non Sig. at $\mathrm{P}>0.05$; Testing based on repeated Measurement test; Com. : Combination of all probable pair wised.

Assessments Intervals Scoring Scales of Relative Sufficiency Coefficient (RS\%): [L: Low $(0.00-33.3)]$; [M: Moderate (33.3 - 66.7)]; [H: High (66.7 - 100)]

Table (3) shows a summary statistics of knowledge concerning in management of third degree heart block Results of testing significant reported highly significant differences at $\mathrm{P}<0.01$ toward impact of applied program through raising knowledge 
grades of studied respondents at post1 period and the results shows the level of comparsion was significant in all items of study group while non significant in all control grouop except in (third degree heart block is alethal dysrhythmia because it may progress to) that could be enable to confirms importance and successfulness of applying the proposed program The results of the controlled group has recorded completely immovable responses over the three periods of times with low level of assessed.

Table (4): Descriptive Statistics of the studied groups according to (Nurses knowledge concerning in management of Bundle branch Block (BBB) along studied periods with comparisons significant

\begin{tabular}{|c|c|c|c|c|c|c|c|c|c|c|c|c|c|c|}
\hline \multirow[b]{2}{*}{$\begin{array}{c}\text { Nurses knowledge } \\
\text { concerning in } \\
\text { management of Bundle } \\
\text { branch block (BB) }\end{array}$} & \multirow[b]{2}{*}{$\begin{array}{c}\text { Perio } \\
\text { d }\end{array}$} & \multirow[b]{2}{*}{ No. } & \multicolumn{6}{|c|}{ Study } & \multicolumn{6}{|c|}{ Control } \\
\hline & & & $\begin{array}{l}\mathbf{M} \\
\mathbf{S}\end{array}$ & $\begin{array}{l}\text { S } \\
\text { D }\end{array}$ & $\begin{array}{l}\mathbf{R} \\
\mathbf{S} \\
\%\end{array}$ & $\begin{array}{l}\text { A } \\
\mathbf{S} \\
\text { S. }\end{array}$ & $\begin{array}{l}\mathbf{C} \\
\mathbf{o} \\
\mathbf{m} \\
\text {. }\end{array}$ & $\begin{array}{l}\text { C. } \\
\text { S. }\end{array}$ & $\begin{array}{c}\mathbf{M} \\
\mathbf{S}\end{array}$ & $\begin{array}{l}\text { S } \\
\mathbf{D}\end{array}$ & $\begin{array}{l}\mathbf{R} \\
\mathbf{S} \\
\%\end{array}$ & $\begin{array}{l}\text { A } \\
\text { SS } \\
\text {. }\end{array}$ & $\begin{array}{l}\text { C } \\
\mathbf{o} \\
\mathbf{m}\end{array}$ & $\begin{array}{c}\text { C.S } \\
.\end{array}$ \\
\hline Bundle branch block & Pre & 40 & $\begin{array}{l}1 . \\
7 \\
5\end{array}$ & $\begin{array}{c}0 . \\
84\end{array}$ & $\begin{array}{c}58 \\
.3\end{array}$ & $\mathbf{M}$ & $\begin{array}{l}1 \\
\mathbf{X} \\
2\end{array}$ & $\begin{array}{c}\mathbf{H} \\
\mathbf{S}\end{array}$ & $\begin{array}{l}1 . \\
68\end{array}$ & $\begin{array}{l}0 . \\
83\end{array}$ & $\begin{array}{l}5 \\
6 \\
\\
\end{array}$ & $\mathbf{M}$ & $\begin{array}{l}1 \\
\mathbf{X} \\
2\end{array}$ & NS \\
\hline $\begin{array}{l}\text { is an interruption in the } \\
\text { cardiac electrical } \\
\text { conduction system of }\end{array}$ & Post1 & 40 & $\begin{array}{l}2 . \\
6 \\
3\end{array}$ & $\begin{array}{c}0 . \\
59\end{array}$ & $\begin{array}{r}87 \\
.7\end{array}$ & $\mathbf{H}$ & $\begin{array}{l}\mathbf{1} \\
\mathbf{X} \\
\mathbf{3}\end{array}$ & $\begin{array}{c}\mathbf{H} \\
\mathbf{S}\end{array}$ & $\begin{array}{l}1 . \\
78\end{array}$ & $\begin{array}{l}0 . \\
89\end{array}$ & $\begin{array}{l}5 \\
9 \\
\dot{3}\end{array}$ & $\mathbf{M}$ & $\begin{array}{l}\mathbf{1} \\
\mathbf{X} \\
\mathbf{3}\end{array}$ & HS \\
\hline both & Post2 & 40 & $\begin{array}{l}2 . \\
7 \\
0\end{array}$ & $\begin{array}{l}0 . \\
46\end{array}$ & $\begin{array}{l}90 \\
.0\end{array}$ & $\mathbf{H}$ & $\begin{array}{l}2 \\
\mathbf{X} \\
\mathbf{3}\end{array}$ & $\begin{array}{l}\mathbf{N} \\
\mathbf{S}\end{array}$ & $\begin{array}{l}1 . \\
90\end{array}$ & $\begin{array}{l}0 . \\
84\end{array}$ & $\begin{array}{l}6 \\
3 \\
\\
3\end{array}$ & $\mathbf{M}$ & $\begin{array}{l}2 \\
\mathbf{X} \\
\mathbf{3}\end{array}$ & $\mathbf{S}$ \\
\hline \multirow{3}{*}{$\begin{array}{l}\text { With Bundle branch block } \\
\text { the electrical impulses will } \\
\text { takes more time to travel }\end{array}$} & Pre & 40 & $\begin{array}{l}1 . \\
9 \\
8\end{array}$ & $\begin{array}{l}0 . \\
92\end{array}$ & $\begin{array}{r}66 \\
.0\end{array}$ & $\mathbf{M}$ & $\begin{array}{l}1 \\
\mathbf{X} \\
\mathbf{2}\end{array}$ & $\begin{array}{l}\mathbf{N} \\
\mathbf{S}\end{array}$ & $\begin{array}{l}1 . \\
70\end{array}$ & $\begin{array}{l}0 . \\
85\end{array}$ & $\begin{array}{l}5 \\
6 \\
\\
\end{array}$ & $\mathbf{M}$ & $\begin{array}{l}1 \\
\mathbf{X} \\
2\end{array}$ & NS \\
\hline & Post1 & 40 & $\begin{array}{l}2 . \\
2 \\
5\end{array}$ & $\begin{array}{l}0 . \\
63\end{array}$ & $\begin{array}{r}75 \\
.0\end{array}$ & $\mathbf{H}$ & $\begin{array}{l}\mathbf{1} \\
\mathbf{X} \\
\mathbf{3}\end{array}$ & $\mathbf{S}$ & $\begin{array}{l}1 . \\
88\end{array}$ & $\begin{array}{l}0 . \\
91\end{array}$ & $\begin{array}{l}6 \\
2 \\
\end{array}$ & M & $\begin{array}{l}\mathbf{1} \\
\mathbf{X} \\
\mathbf{3}\end{array}$ & NS \\
\hline & Post2 & 40 & $\begin{array}{c}2 . \\
3 \\
8\end{array}$ & $\begin{array}{l}0 . \\
49\end{array}$ & $\begin{array}{r}79 \\
.3\end{array}$ & $\mathbf{H}$ & $\begin{array}{l}\mathbf{2} \\
\mathbf{X} \\
\mathbf{3}\end{array}$ & $\mathbf{S}$ & $\begin{array}{l}2 . \\
15\end{array}$ & $\begin{array}{l}0 . \\
80\end{array}$ & $\begin{array}{l}7 \\
\mathbf{1} \\
. \\
7\end{array}$ & $\mathbf{H}$ & $\begin{array}{c}\mathbf{2} \\
\mathbf{X} \\
\mathbf{3}\end{array}$ & NS \\
\hline \multirow{3}{*}{$\begin{array}{c}\text { The QRS compelx usually } \\
\text { narrowing than normal } \\
\text { usually less than } 0.12 \\
\text { second }\end{array}$} & Pre & 40 & $\begin{array}{l}1 . \\
6 \\
3\end{array}$ & $\begin{array}{c}0 . \\
77\end{array}$ & $\begin{array}{c}54 \\
.3\end{array}$ & $\mathbf{M}$ & $\begin{array}{l}1 \\
\mathbf{X} \\
\mathbf{2}\end{array}$ & $\begin{array}{c}\mathbf{H} \\
\mathbf{S}\end{array}$ & $\begin{array}{l}1 . \\
73\end{array}$ & $\begin{array}{l}0 . \\
88\end{array}$ & $\begin{array}{l}5 \\
7 \\
. \\
7\end{array}$ & $\mathbf{M}$ & $\begin{array}{l}\mathbf{1} \\
\mathbf{X} \\
\mathbf{2}\end{array}$ & NS \\
\hline & Post1 & 40 & $\begin{array}{c}2 . \\
2 \\
8\end{array}$ & $\begin{array}{l}0 . \\
64\end{array}$ & $\begin{array}{c}76 \\
.0\end{array}$ & $\mathbf{H}$ & $\begin{array}{l}\mathbf{1} \\
\mathbf{X} \\
\mathbf{3}\end{array}$ & $\begin{array}{l}\mathbf{H} \\
\mathbf{S}\end{array}$ & $\begin{array}{l}1 . \\
88\end{array}$ & $\begin{array}{l}0 . \\
91\end{array}$ & $\begin{array}{l}6 \\
2 \\
. \\
7\end{array}$ & $\mathbf{M}$ & $\begin{array}{c}\mathbf{1} \\
\mathbf{X} \\
\mathbf{3}\end{array}$ & HS \\
\hline & Post2 & 40 & $\begin{array}{c}2 . \\
5 \\
8\end{array}$ & $\begin{array}{c}\text { 0. } \\
50\end{array}$ & $\begin{array}{r}86 \\
.0\end{array}$ & $\mathbf{H}$ & $\begin{array}{l}\mathbf{2} \\
\mathbf{X} \\
\mathbf{3}\end{array}$ & $\begin{array}{l}\mathbf{H} \\
\mathbf{S}\end{array}$ & $\begin{array}{l}2 . \\
10\end{array}$ & $\begin{array}{c}0 . \\
78\end{array}$ & $\begin{array}{l}7 \\
\mathbf{0} \\
\\
\mathbf{0}\end{array}$ & $\mathbf{H}$ & $\begin{array}{l}\mathbf{2} \\
\mathbf{X} \\
\mathbf{3}\end{array}$ & HS \\
\hline
\end{tabular}




\begin{tabular}{|c|c|c|c|c|c|c|c|c|c|c|c|c|c|c|}
\hline \multirow{3}{*}{$\begin{array}{c}\text { If both Bundle branches } \\
\text { are blocked the electrical } \\
\text { impulses will reach both } \\
\text { ventricles later than } \\
\text { normal }\end{array}$} & Pre & 40 & $\begin{array}{l}1 . \\
8 \\
3\end{array}$ & $\begin{array}{c}\text { 0. } \\
87\end{array}$ & $\begin{array}{c}61 \\
.0\end{array}$ & $\mathbf{M}$ & $\begin{array}{l}\mathbf{1} \\
\mathbf{X} \\
\mathbf{2}\end{array}$ & $\begin{array}{l}\mathbf{H} \\
\mathbf{S}\end{array}$ & $\begin{array}{l}1 . \\
75\end{array}$ & $\begin{array}{c}\text { 0. } \\
84\end{array}$ & $\begin{array}{l}5 \\
8 \\
\dot{3}\end{array}$ & $\mathbf{M}$ & $\begin{array}{l}1 \\
X \\
\mathbf{2}\end{array}$ & NS \\
\hline & Post1 & 40 & $\begin{array}{c}2 . \\
3 \\
5\end{array}$ & $\begin{array}{c}0 . \\
58\end{array}$ & $\begin{array}{c}78 \\
.3\end{array}$ & $\mathbf{H}$ & $\begin{array}{l}\mathbf{1} \\
\mathbf{X} \\
\mathbf{3}\end{array}$ & $\begin{array}{l}\mathbf{H} \\
\mathbf{S}\end{array}$ & $\begin{array}{l}1 . \\
90\end{array}$ & $\begin{array}{c}\text { 0. } \\
87\end{array}$ & $\begin{array}{l}6 \\
3 \\
3 \\
3\end{array}$ & $\mathbf{M}$ & $\begin{array}{l}\mathbf{1} \\
\mathbf{X} \\
\mathbf{3}\end{array}$ & HS \\
\hline & Post2 & 40 & $\begin{array}{c}2 . \\
4 \\
7\end{array}$ & $\begin{array}{c}0 . \\
51\end{array}$ & $\begin{array}{r}82 \\
.3\end{array}$ & $\mathbf{H}$ & $\begin{array}{c}\mathbf{2} \\
\mathbf{X} \\
\mathbf{3}\end{array}$ & $\begin{array}{l}\mathbf{N} \\
\mathbf{S}\end{array}$ & 2. & $\begin{array}{c}0 . \\
58\end{array}$ & $\begin{array}{l}7 \\
4 \\
\dot{3}\end{array}$ & H & $\begin{array}{l}\mathbf{2} \\
\mathbf{X} \\
\mathbf{3}\end{array}$ & HS \\
\hline
\end{tabular}

${ }^{(*)}$ HS: Highly Sig. at $\mathrm{P}<0.01$; S: Sig. at $\mathrm{P}<0.05$; NS: Non Sig. at $\mathrm{P}>0.05$; Testing based on repeated Measurement test; Com. : Combination of all probable pair wised . Assessments Intervals Scoring Scales of Relative Sufficiency Coefficient (RS\%): [L: Low (33.3 - 55.5)]; [M: Moderate (55.5 - 77.7)]; [H: High (77.7 - 100)].

Table (4) reported highly significant program. The results of the control group has differences at $\mathrm{P}<0.01$ toward impact of applied illustrated marginal level of improvements program through raising knowledge grades of grade over the three periods of times with a studied respondents at post 1 period, and that moderate and high level of assessed, since could be enable to confirms importance and studied subjects hasprecedingassessedinitially. successfulness of applying the proposed

Table (5): Descriptive Statistics of the studied groups according to (Nurses knowledge concerning in drugs and pacemaker used in management of AV heart block (Pm)) along studied periods with comparisons significant

\begin{tabular}{|c|c|c|c|c|c|c|c|c|c|c|c|c|c|c|}
\hline \multirow{2}{*}{$\begin{array}{l}\text { Nurses knowledge } \\
\text { concerning in drugs } \\
\text { \& pace-maker used } \\
\text { in management of } \\
\text { AV heart block } \\
(\mathrm{Pm})\end{array}$} & \multirow[b]{2}{*}{ Period } & \multirow[b]{2}{*}{$\begin{array}{l}\text { N } \\
\text { o. }\end{array}$} & \multicolumn{6}{|c|}{ Study } & \multicolumn{6}{|c|}{ Control } \\
\hline & & & MS & SD & $\begin{array}{l}\mathbf{R} \\
\mathbf{S} \\
\mathbf{\%}\end{array}$ & $\begin{array}{c}\text { As } \\
\text { s. }\end{array}$ & $\begin{array}{c}\mathbf{C} \\
\mathbf{o} \\
\mathbf{m}\end{array}$ & $\begin{array}{l}\text { C. } \\
\text { S. }\end{array}$ & $\begin{array}{c}\mathbf{M} \\
\mathbf{S}\end{array}$ & $\begin{array}{l}\text { S } \\
\text { D }\end{array}$ & $\begin{array}{c}\text { RS } \\
\%\end{array}$ & $\begin{array}{c}\text { A } \\
\text { ss. }\end{array}$ & $\begin{array}{c}\text { C } \\
\text { o } \\
\text { m }\end{array}$ & $\begin{array}{c}\text { C.S } \\
.\end{array}$ \\
\hline \multirow{3}{*}{$\begin{array}{c}\text { In mobitz II AV } \\
\text { heart block } \\
\text { dopamine may be } \\
\text { administer for } \\
\text { systole blood } \\
\text { pressure less than }\end{array}$} & Pre & 40 & 0.30 & 0.46 & \begin{tabular}{|c|}
$\mathbf{3 0}$ \\
.0
\end{tabular} & M & $\begin{array}{l}1 \\
X \\
2\end{array}$ & $\begin{array}{l}\mathbf{H} \\
\mathbf{S}\end{array}$ & $\begin{array}{c}0 . \\
18\end{array}$ & $\begin{array}{l}\mathbf{0} \\
\dot{3} \\
8\end{array}$ & $\begin{array}{c}18 . \\
0\end{array}$ & $\mathbf{L}$ & $\begin{array}{l}1 \\
X \\
2\end{array}$ & NS \\
\hline & Post1 & 40 & 0.78 & 0.42 & \begin{tabular}{|c}
78 \\
.0
\end{tabular} & $\mathbf{H}$ & $\begin{array}{l}\mathbf{1} \\
\mathbf{X} \\
\mathbf{3}\end{array}$ & $\begin{array}{l}\mathbf{H} \\
\mathbf{S}\end{array}$ & $\begin{array}{c}\text { 0. } \\
18\end{array}$ & $\begin{array}{l}0 \\
\dot{3} \\
8\end{array}$ & $\begin{array}{c}18 . \\
0\end{array}$ & $\mathbf{L}$ & $\begin{array}{l}1 \\
\mathbf{X} \\
\mathbf{3}\end{array}$ & $\mathbf{S}$ \\
\hline & Post2 & 40 & 0.92 & 0.27 & $\begin{array}{c}92 \\
.0\end{array}$ & H & $\begin{array}{l}\mathbf{2} \\
\mathbf{X} \\
\mathbf{3}\end{array}$ & $\mathbf{S}$ & $\begin{array}{c}\text { 0. } \\
30\end{array}$ & $\begin{array}{l}0 \\
\dot{4} \\
6\end{array}$ & $\begin{array}{c}30 . \\
\text { 0 }\end{array}$ & $\mathbf{L}$ & $\begin{array}{l}\mathbf{2} \\
\mathbf{X} \\
\mathbf{3}\end{array}$ & $\mathbf{S}$ \\
\hline
\end{tabular}


Mosul Journal of Nursing, Vol. 9, No. 1, 2021 ( 8-20 )

\begin{tabular}{|c|c|c|c|c|c|c|c|c|c|c|c|c|c|c|}
\hline \multirow{3}{*}{$\begin{array}{c}\text { Epinephrin } \\
\text { administer to } \\
\text { patients who do not } \\
\text { respond to }\end{array}$} & Pre & 40 & 0.22 & 0.42 & $\begin{array}{c}22 \\
.0\end{array}$ & $\mathbf{M}$ & $\begin{array}{l}1 \\
X \\
2\end{array}$ & $\begin{array}{c}\mathbf{H} \\
\mathbf{S}\end{array}$ & $\begin{array}{c}0 . \\
20\end{array}$ & $\begin{array}{l}\mathbf{0} \\
\dot{4} \\
1\end{array}$ & $\begin{array}{c}20 . \\
0\end{array}$ & & $\begin{array}{l}1 \\
X \\
2\end{array}$ & NS \\
\hline & Post1 & 40 & 0.80 & 0.41 & $\begin{array}{c}80 \\
.0\end{array}$ & $\mathbf{H}$ & $\begin{array}{l}1 \\
\mathbf{X} \\
\mathbf{3}\end{array}$ & $\begin{array}{c}\mathbf{H} \\
\mathbf{S}\end{array}$ & $\begin{array}{c}0 . \\
23\end{array}$ & $\begin{array}{l}\mathbf{0} \\
4 \\
2\end{array}$ & $\begin{array}{c}23 . \\
0\end{array}$ & $\mathbf{L}$ & $\begin{array}{l}1 \\
\mathbf{X} \\
\mathbf{3}\end{array}$ & $\mathbf{S}$ \\
\hline & Post2 & 40 & 0.95 & 0.22 & $\begin{array}{c}95 \\
.0\end{array}$ & $\mathbf{H}$ & $\begin{array}{l}2 \\
X \\
3\end{array}$ & $\mathbf{S}$ & $\begin{array}{c}\text { 0. } \\
32\end{array}$ & $\begin{array}{l}0 \\
\dot{4} \\
7\end{array}$ & $\begin{array}{c}32 . \\
0\end{array}$ & $\mathbf{L}$ & $\begin{array}{l}2 \\
X \\
3\end{array}$ & $\mathbf{S}$ \\
\hline \multirow{3}{*}{$\begin{array}{l}\text { In second and third } \\
\text { heart block the type } \\
\text { of pacemaker is }\end{array}$} & Pre & 40 & 0.32 & 0.47 & $\begin{array}{c}32 \\
.0\end{array}$ & $\mathbf{M}$ & $\begin{array}{l}1 \\
X \\
2\end{array}$ & $\begin{array}{c}\mathbf{H} \\
\mathbf{S}\end{array}$ & $\begin{array}{c}\text { 0. } \\
30\end{array}$ & $\begin{array}{l}0 \\
\dot{0} \\
4 \\
6\end{array}$ & $\begin{array}{c}30 . \\
0\end{array}$ & $\mathbf{L}$ & $\begin{array}{l}1 \\
X \\
2\end{array}$ & $\mathbf{S}$ \\
\hline & Post1 & 40 & 0.75 & 0.44 & $\begin{array}{c}75 \\
.0\end{array}$ & $\mathbf{H}$ & $\begin{array}{l}1 \\
X \\
3\end{array}$ & $\begin{array}{c}\mathbf{H} \\
\mathbf{S}\end{array}$ & $\begin{array}{c}0 . \\
15\end{array}$ & $\begin{array}{l}0 \\
\dot{3} \\
6\end{array}$ & $\begin{array}{c}15 . \\
0\end{array}$ & $\mathbf{L}$ & $\begin{array}{l}1 \\
\mathbf{X} \\
\mathbf{3}\end{array}$ & NS \\
\hline & Post2 & 40 & 0.83 & 0.38 & $\begin{array}{c}83 \\
.0\end{array}$ & $\mathbf{H}$ & $\begin{array}{l}2 \\
X \\
3\end{array}$ & $\begin{array}{l}\mathbf{N} \\
\mathbf{S}\end{array}$ & $\begin{array}{c}0 . \\
25\end{array}$ & $\begin{array}{l}0 \\
\dot{4} \\
4\end{array}$ & $\begin{array}{c}25 . \\
0\end{array}$ & $\mathbf{L}$ & $\begin{array}{l}2 \\
X \\
3\end{array}$ & $\mathbf{S}$ \\
\hline \multirow{3}{*}{$\begin{array}{c}\text { Pacemaker is a } \\
\text { small battery - } \\
\text { operated device that } \\
\text { initiate electrical } \\
\text { impulse in }\end{array}$} & Pre & 40 & 0.30 & 0.46 & $\begin{array}{c}30 \\
.0\end{array}$ & $\mathbf{M}$ & $\begin{array}{l}1 \\
X \\
2\end{array}$ & $\begin{array}{l}\mathbf{H} \\
\mathbf{S}\end{array}$ & $\begin{array}{c}0 . \\
45\end{array}$ & $\begin{array}{l}0 \\
\dot{5} \\
0\end{array}$ & $\begin{array}{c}45 . \\
0\end{array}$ & M & $\begin{array}{l}1 \\
X \\
2\end{array}$ & NS \\
\hline & Post1 & 40 & 0.65 & 0.48 & $\begin{array}{c}65 \\
.0\end{array}$ & $\mathbf{H}$ & $\begin{array}{l}1 \\
X \\
3\end{array}$ & $\begin{array}{l}\mathbf{H} \\
\mathbf{S}\end{array}$ & $\begin{array}{c}0 . \\
45\end{array}$ & $\begin{array}{l}\mathbf{0} \\
\dot{5} \\
\mathbf{0}\end{array}$ & $\begin{array}{c}45 . \\
0\end{array}$ & $\mathbf{M}$ & $\begin{array}{l}1 \\
\mathbf{X} \\
\mathbf{3}\end{array}$ & NS \\
\hline & Post2 & 40 & 0.72 & 0.45 & $\begin{array}{c}72 \\
.0\end{array}$ & $\mathbf{H}$ & $\begin{array}{l}2 \\
X \\
3\end{array}$ & $\begin{array}{l}\mathbf{N} \\
\mathbf{S}\end{array}$ & $\begin{array}{c}0 . \\
52\end{array}$ & $\begin{array}{l}0 \\
\\
5 \\
1\end{array}$ & $\begin{array}{c}52 . \\
0\end{array}$ & $\mathbf{M}$ & $\begin{array}{l}2 \\
X \\
3\end{array}$ & NS \\
\hline \multirow{3}{*}{$\begin{array}{c}\text { Fixed pacemaker set } \\
\text { to generate impulses } \\
\text { at a constant rate } \\
\text { usually }\end{array}$} & Pre & 40 & 0.22 & 0.42 & $\begin{array}{c}22 \\
.0\end{array}$ & $\mathbf{M}$ & $\begin{array}{l}1 \\
X \\
2\end{array}$ & $\begin{array}{l}\mathbf{H} \\
\mathbf{S}\end{array}$ & $\begin{array}{l}0 . \\
22\end{array}$ & $\begin{array}{l}\mathbf{0} \\
\dot{4} \\
2\end{array}$ & $\begin{array}{c}22 . \\
0\end{array}$ & $\mathbf{L}$ & $\begin{array}{l}1 \\
X \\
2\end{array}$ & HS \\
\hline & Post1 & 40 & 0.78 & 0.42 & $\begin{array}{c}78 \\
.0\end{array}$ & $\mathbf{H}$ & $\begin{array}{l}1 \\
\mathbf{X} \\
\mathbf{3}\end{array}$ & $\begin{array}{l}\mathbf{H} \\
\mathbf{S}\end{array}$ & $\begin{array}{c}\text { 0. } \\
03\end{array}$ & $\begin{array}{l}0 \\
\dot{1} \\
6\end{array}$ & 2.5 & $\mathbf{L}$ & $\begin{array}{l}1 \\
\mathbf{X} \\
\mathbf{3}\end{array}$ & NS \\
\hline & Post2 & 40 & 0.88 & 0.33 & $\begin{array}{c}88 \\
.0\end{array}$ & $\mathbf{H}$ & $\begin{array}{l}2 \\
\mathbf{X} \\
\mathbf{3}\end{array}$ & $\mathbf{S}$ & $\begin{array}{c}0 . \\
20\end{array}$ & $\begin{array}{l}0 \\
4 \\
4\end{array}$ & $\begin{array}{c}20 . \\
0\end{array}$ & $\mathbf{L}$ & $\begin{array}{l}\mathbf{2} \\
\mathbf{X} \\
\mathbf{3}\end{array}$ & HS \\
\hline
\end{tabular}

${ }^{*}$ HS: Highly Sig. at $\mathrm{P}<0.01$; S: Sig. at $\mathrm{P}<0.05$; NS: Non Sig. at $\mathrm{P}>0.05$; Testing based on repeated Measurement test; Com. : Combination of all probable pair wised. Assessments 


\section{Mosul Journal of Nursing, Vol. 9, No. 1, 2021 ( 8-20 )}

\section{Intervals Scoring Scales of Relative Sufficiency Coefficient (RS\%): [L: Low (0.00 - 33.3)]; [M: Moderate (33.3 - 66.7)]; [H: High $(66.7-100)]$.}

Table (5) reported highly significant differences at $\mathrm{P}<0.01$ toward impact of applied program through raising knowledge grades of studied respondents at post 1 period, and that could be enable to confirms importance and successfulness of applying the proposed program. In addition to that, and rather than testing significant are too sensitive to improvements that occurred for repeated measurements statistic in the study group, but all of studied items are illustrated too highly and meaningful changeability with highly levels of assessments along pre-postlperiods concerning, as well as post 2 period does required to studied process of application proposed of an educational program indeed, since supplementary improvements are accounted, and the observed outcomes were coverage to the greatest level of approved scale. The results of the controlled group has recorded completely immovable responses over the three periods of times with low level of assessed.

Table(6): Relationships (Analysis of Covariance) concerning Compliance Regarding to Life Style Modification in the study group and SDCv.

\begin{tabular}{|c|c|c|c|c|c|c|c|}
\hline Group & $\begin{array}{l}\text { Source of } \\
\text { Variations }\end{array}$ & $\begin{array}{c}\text { Type III Sum } \\
\text { of Squares }\end{array}$ & d.f. & $\begin{array}{c}\text { Mean } \\
\text { Square }\end{array}$ & $\begin{array}{c}\mathbf{F} \\
\text { Statistic } \\
\end{array}$ & $\begin{array}{c}\text { Sig. } \\
\text { Levels }\end{array}$ & C.S. ${ }^{(*)}$ \\
\hline \multirow{8}{*}{ Study } & Intercept & $4 \mathbf{4 3 2 0 5 . 1 5}$ & $\overline{11}$ & $4 \quad 43205$ & 891.0 & $\begin{array}{l}0.000 \\
\end{array}$ & HS \\
\hline & Gender & 2.180 & 1 & 2.180 & 0.045 & 0.834 & NS \\
\hline & Age Group & 90.90 & 3 & 30.30 & 0.625 & 0.605 & NS \\
\hline & Residency & 168.20 & 1 & 168.20 & 3.469 & 0.072 & NS \\
\hline & Marital Status & 2.160 & 2 & 1.082 & 0.022 & 0.978 & NS \\
\hline & Education Levels & 203.19 & 2 & 101.60 & 2.095 & 0.141 & NS \\
\hline & Error & 1454.71 & 30 & 48.49 & \multirow{2}{*}{\multicolumn{3}{|c|}{ R-Squared $=0.281$}} \\
\hline & Total & 282523 & 40 & & & & \\
\hline
\end{tabular}

\section{${ }^{(*)}$ HS: Highly Sig. at $\mathrm{P}<0.01$; Non Sig. at $\mathrm{P}>0.05$; Statistical hypothesis based on Analysis of Covariance (ANCOVA).}

Results shows that weak relationships are a proved with (SDCv.), since no significant relationships were accounted at $\mathrm{P}>0.05$, and according to that it could be concludes that studied questionnaire of nurse's knowledge concerning management for patients with heart block improvements through applying the suggested of an educational program could be generalize on the studied population even though differences within their socio-demographical characteristics variables of studied subjects.

\section{Discussion:}

Throughout the course of data analysis of sample (40) nurse, the results shows 23 ( $57.5 \%)$ were females and $17(42.5 \%)$ were males. These finding is agrement with the result of Ruhwanya and others (2018) who find More than three quarters of participants were females $(79.4 \%)$.

Also the the results shows the most of the nurses at age between (20-29 years) and constitute $26(65.0 \%)$

But these findings disagree with the study of Mohamed and others (2016) in Egypt, who reported that More than one third of the study sample aged between 40 to 49 years.

With regard to resideance $28(70.0 \%)$ were living in urban areas.Explanation of this result related to situation of hospitals at center of governorate there fore the 


\section{Mosul Journal of Nursing, Vol. 9, No. 1, 2021 ( 8-20 )}

researchrer find most of nurses from urban area. But these findings disagree with the study in Egypt by Mohamed and others (2016) in Egypt, who reported that more than half of the sample (62\%), were living in rural areas

Most of the nurses were single and constituted $26(65.0 \%)$.also the result shows high percentage from nurses were complete bachelor of nursing and more and constitute 20(50.0\%). Respect to subjects of studied (SDCv.), results shows that studied groups recorded no significant differences at $\mathrm{P}>0.05$

Our study has revealed in table (2) the majority of studied subjects are worked at CCU unit, since they are accounted $15(37.5 \%)$, and $14(35.0 \%)$ in the study and controlled groups respectively as well as the most of training named concerned with the studied subjects previously was at CCU unit, since they are accounted 21(52.5\%), and $22(55.0 \%)$ in the study and controlled groups respectively.

The explanation of this result because of the nurses worked at this site usually more faced like this cases (heart block ) there for need for more knowldge program about care of patients with arrhythemia there for find most of nurses from this site. This suggestion is in agreement with the finding obtained from Kingwood (2012) who has mentioned that education programs have been designed to keep nurses up to date with current practices and trends. They ensure nursing staff' abilities through mandatory annual competency sessions .

In relative to the nurses knowldge to ward management third degree heart block the result shows in table (6) Results refers to significant with reference of studied items, as well as scoring scales assessments concerning effectiveness of applying an educational program were reported highly significant differences at $\mathrm{P}<0.01$ toward impact of applied program through raising knowledge grades of studied respondents while the cntrol group shows poor a ssessment of knowldg in all study level Interpret of this result according to the researcher openion related to impact of an education program on nurses knowldge toward third degree heart block confirms importance and successfulness of applying this proposed program there for the results appeare low significant for pre test while the result range from medera to high significant for both post 1 and post2 with regard to the control group the results range from low to moderate assessment during pre and post 1 ,post

These findings agrees with study of Ruhwanya and others (2018) ) stated that the resuls is, a low knowledge score was noted regarding complete heart block in the ECG strip, with only $38.3 \%$ of participants being able to identify it correctly

Also other results confirmed by Schultz (2010) suggested that hospital administrators should encourage nurses to 


\section{Mosul Journal of Nursing, Vol. 9, No. 1, 2021 ( 8-20 )}

participate in a clinical practice development programme, to increase their knowledge and skills in arrhythmia monitoring. This can encourage nurses to enjoy learning, which makes it easier for them to master the skill.

With regard to the nurses knowldge about Bundle Branch Block which shows at table (7) the results of testing significant with reference of studied items, as well as scoring scales assessments concerning effectiveness of applying an educational program were reported highly significant differences at $\mathrm{P}<0.01$ toward impact of applied program through raising knowledge grades of studied respondents at post1 period, and that could be enable to confirms importance and successfulness of applying the proposed program while the results of the control group has illustrated marginal level of improvements grade over the three periods of times with a moderate and high level of assessed, since studied subjects has preceding assessed initially.

Our study finding is agreed with a similar study done by Nursing Patient - Cantered Collaborative Care (2013) who reported that more than two third of nurses, their knowledge regarding bundle branch block was unsatisfactory.

The result in table (5) about Nurses knowledge concerning in drugs and pacemaker used in management of $\mathrm{AV}$ heart block revealed that there is highly significant differences at $\mathrm{P}<0.01$ toward impact of applied program through raising knowledge grades of studied respondents at post1 period, and that could be enable to confirms importance and successfulness of applying the proposed program .With regard to the conrol group the results shows completely immovable responses over the three periods of times with low level of assessed.

As well, our finding is consistent with another similar study done by Ayad and others (2016) who assessed the critical care nurses' knowledge related to management of cardiac dysrhythmias by drugs and pacemaker at Benha University Hospital and find that majority of the nurses were having an unsatisfactory knowledge about cardiac dysrhythmias management . Reference: -

-American College of Cardiology Foundation. (J Am Coll Cardiol EP 2016;2:181-92) 2016.

- Ayad M, Mousa M, Haneen A, et al. Kufa J Nurs Sci. 6(2), (2016).

-Crisel RK, Farzaneh-Far R, Na B, Whooley MA. First-degree atrioventricular block is associated with heart failure and death in persons with stable coronary artery disease: data from the Heart and Soul Study. Eur Heart J. 2011;32:1875-1880.

-Chow GV, Marine JE, Fleg JL. Epidemiology of arrhythmias and conduction disorders in older adults. Clin Geriatr Med. 2012;28:539553.

-Dake,SH ; Dias, R ,.Effectiveness of Information Booklet regarding Home Care of Pacemaker among the Patients with Complete Heart Block.International Journal of Science and Research (IJSR) (2012): ISSN (Online): 2319-7064 Impact Factor :3.358:PP:1877-1883

-Kingwood (Campus)Certificate III in (NRT)Health Services Assistance (Assisting in Nursing Work in Acute Care)Course no. 17596Training Package/s: CHC02 Community Services National Course Code:2012:HLT32507Course 


\section{Mosul Journal of Nursing, Vol. 9, No. 1, 2021 ( 8-20 )}

-Mohamed, A , Shreif, W ,Mohamed ,H and Maaty,A Effectiveness of Educational Program on Knowledge and Practice of Patients Undergoing Permanent Pacemaker. Journal of Nursing and Health Science (IOSRJNHS) ISSN: 2320-1940 Volume 5, Issue 6 Ver. VI (Nov. - Dec. 2016), PP 72-83.

- Nursing Patient - Cantered Collaborative Care (7th ed). Elsevier Saunders. 730-750 (2013).

- Reynolds , G ,. Managing atrioventricular blocks, ANA Journal . 2014 ,.(9) 1:PP:5 -6.

-Rathore SS, Gersh BJ, Berger PB, Weinfut $\mathrm{KP}$, Octgen WJ, Schulman KA, et al. Acute myocardial infarction complicated by heart block in the elderly: prevalence and outcomes. Am Heart J 2015; 141:47-54.

- Ruhwanya ,D ,.Tarimo, E and Ndile ,M,. Life threatening arrhythmias: Knowledge and skills among nurses working in critical care settings at Muhimbili National Hospital, Dar es Salaam, Tanzania, Tanzania Journal of Health Research, Volume 20, Number 2, April 2018 :PP.17-19.

-Schultz, S.J. (2010) Dysrhythmia Monitoring Practices of Nurses on a Teletry Unit. Teletry Unit. Florida, Florida Brooks College of Health.

- Stewart, A.; Jane, V.; \& Sheehan, M.: Permanent Pacemakers: TheNurse's Role in Patient Education and Follow-up Care, Journal ofCardiovascular Nursing, (2011) ,5(3), Retrieved from:http://journals.lww.com/jcnjournal/Abstr act/1991/04000/Permanent_pacemakers_The_ nurse_s_role_in_pa tient.6.aspx.

- World health organization (WHO). Coronary Heart Disease /selecting cause of death rate per 100.000 (2011) available at http://www.worldlifeexpectancy.com/cause-ofdeath/coronaryheart-disease/by-country. 